\section{P42 THE IMPACT OF A CATERING INITIATIVE IN DETERMINING FOOD CHOICES AND SALT INTAKE IN THE PUBLIC SECTOR}

doi:10.1136/jech.2010.120477.42

F Geaney, J Harrington, I J Perry. Department of Epidemiology and Public Health, University College Cork, cork, Ireland

Background The epidemic of obesity and diet-related chronic diseases are due to a considerable increase in energy intake from sugar, fat and salt in addition to a decreasing level of physical activity. Due to changes in modern lifestyles, individuals now depend on out-of-home eating. There is evidence that the catering sector can have a pivotal role in influencing our food choices.

Objective To examine whether a structured catering initiative could significantly determine food choice and salt intake in the public sector.

Design A cross-sectional comparison study in two hospitals, one of which had implemented a catering initiative focused on reducing sugar, salt and fat intakes.

Setting Two public sector hospitals in Cork, Ireland.

Subjects/Methods A total of 100 random participants aged 18-64 years (fifty, intervention and fifty, non-intervention) who consumed at least one main meal in the hospital staff canteen daily. Each respondent was asked to complete one anonymous $24 \mathrm{~h}$ dietary recall and a questionnaire. Food and nutrient analysis was conducted using WISPC) (Weighed Intake Software Program; Tinuviel Software, Warrington, UK).

Results Reported mean intakes of total fat $(p<0.000)$, saturated fat $(p<0.000)$, salt $(p<0.046)$ and total sugars $(p<0.001)$ were significantly lower in the intervention hospital when adjusting for age and gender. In the intervention hospital, $43 \%$ of respondents exceeded the recommended salt intake of $4-6 \mathrm{~g} /$ day vs $57 \%$ of respondents in the non-intervention hospital. Significantly, $72 \%$ of respondents in the intervention hospital vs $42 \%$ in the non-intervention hospital complied with the recommended under-3 daily servings of foods high in fats and sugar (eg, oils, butter and cakes) $(p<0.005)$.

Conclusion A hospital with a structured catering initiative can serve as a supportive environment to aid the determination of nutritious food choices and reduced salt intake. More public health efforts and health policy changes are needed to motivate caterers in the public sector and other industries into developing interventions that cater to a healthy diet.

\section{P43 DIETARY SALT INTAKE IN IRISH ADULTS}

doi:10.1136/jech.2010.120477.43

G Browne, M Loughrey, J Harrington, J Lutomski, T Fitzgerald, I Perry. Department of Epidemiology and Public Health, Brookfield Health Sciences Complex, University College Cork, Cork, Ireland

Objectives To estimate dietary salt intakes in the Irish population, including variation by age, sex, and measures of obesity.

Design Two cross-sectional studies.

Settings and Participants (i) A nationally representative household sample of 10364 adults, 62\% response rate (SLAN study), (ii) samples of adults aged from $18-81$ years $(\mathrm{N}=599)$ recruited from the general population, a worksite screening exercise and third level students (Safefood study).

Intervention In both studies, participants underwent physical measurements including height, weight, and waist circumference and in both studies energy intake, macronutrients and dietary sources of salt were estimated by means of a standard Food Frequency Questionnaire. In the SLAN study, random urine samples were obtained from 1207 men and women aged 45 years and older. In the Safefood study, participants provided one 24-h urine collection. Completeness of urine samples were validated using a biomarker, para-amino benzoic acid (PABA).

Main Outcome Measures Estimates of salt intake in Irish adults by age, gender, and levels of obesity and the proportion of total salt intake associated with major food groups.

Results Using SLAN study, random urine samples corrected for urine volume the estimates (mean (SD), median) for salt intake per day in adults aged over 45 years were as follows: men, $10.3 \mathrm{~g} \mathrm{(5.0)}$, $9.7 \mathrm{~g}$ and women, $7.4 \mathrm{~g}$ (4.2), $7.1 \mathrm{~g}$. Estimated dietary salt intake (mean (SD), median) based on PABA validated $24 \mathrm{~h}$ urine collections was $9.3 \mathrm{~g} /$ day (4.1), $8.5 \mathrm{~g} /$ day with higher intakes in men: $10.4 \mathrm{~g} /$ day (4.3), $9.7 \mathrm{~g} /$ day than in women: $7.4 \mathrm{~g} /$ day $(2.7), 7.1 \mathrm{~g} /$ day. $86 \%$ of men ( $95 \%$ CI $82 \%$ to $90 \%$ ) and $67 \%$ of women $(95 \%$ CI $60 \%$ to $74 \%$ ) consume more than $6 \mathrm{~g}$ salt per day (current tolerable upper limit). Significant variation in salt intake with age was not detected in the PABA validated samples. Dietary salt intake was significantly associated with general and central obesity in both men and women in analyses adjusted for calorie intake. The food groups contributing most to salt intake were cereals, breads, meat, fish and poultry products, which together account for over $50 \%$ of salt intakes.

Conclusions Dietary salt intakes in Irish adults remain high, with the majority of the population exceeding the current tolerable upper limit of $6 \mathrm{~g}$ per day. At the group level, estimates of salt intake based on random urine samples are similar to those derived from validated 24-h urine collections.

\section{Ethnicity}

\section{P44 THE INFLUENCE OF ACCULTURATION ON ETHNIC DIFFERENCES IN OBESITY IN ENGLAND}

doi:10.1136/jech.2010.120477.44

${ }^{1} \mathrm{~N}$ R Smith, ${ }^{2} Y \mathrm{~J}$ Kelly, ${ }^{3} \mathrm{~J}$ Y Nazroo. ${ }^{1}$ Department of Epidemiology \& Population Health, London School of Hygiene and Tropical Medicine, London, UK; ${ }^{2}$ Department of Epidemiology and Public Health, University College London, London, UK; ${ }^{3}$ Department of Sociology, University of Manchester, Manchester, UK

Background Ethnic differences in obesity have been well documented, but comparatively little is known about whether these differences vary according to the degree of exposure to the new environment following migration.

Objectives To investigate the extent of generational differences in adult health-related lifestyles and socioeconomic circumstances, and explore whether these differences might explain changing patterns of obesity in ethnic minorities in England.

Method Seven ethnic minority groups were selected from the ethnically boosted 1999 and 2004 Health Survey for England (Indian $\mathrm{n}=887$; Pakistani $\mathrm{n}=603$; Bangladeshi $\mathrm{n}=275$; Black Caribbean $\mathrm{n}=762$; Black African $\mathrm{n}=147$; Chinese $\mathrm{n}=413$; and Irish $\mathrm{n}=1438$ ). A White group was used as a reference population $(\mathrm{n}=5899)$. Age and sex adjusted logistic regression estimated the odds of having a poor health behaviour in the second generation compared to the first. Age- and sex-adjusted odds of being obese in the second generation compared to the first were estimated before and after adjusting for generational differences in health related behaviours (snacking, eating cakes and fried foods, having low levels of physical exercise, any drinking including binges, current smoking status) and socioeconomic factors (social class, equivalised income and highest qualification)

Results Overall, second generation ethnic minority men and women were significantly more likely than the first to have low vegetable consumption (<one portion/day), snack daily on chocolate, crisps, biscuits and cakes, be current drinkers and binge drink. Second generation women were more likely to currently smoke than the first generation. Conversely, men and women were significantly less likely to have low levels of physical activity in the second generation. However, there were considerable variations in the uptake of 